\title{
Taxonomía y distribución de Nathalis (Lepidoptera: Pieridae) en Colombia
}

\author{
Hannier W. Pulido-B. ${ }^{1}$, M. Gonzalo Andrade-C. ${ }^{2}$ \& Jorge Llorente-Bousquets ${ }^{3 *}$ \\ 1. Instituto de Ciencias Naturales, Universidad Nacional de Colombia, Apartado 7495, Bogotá, D.C., Colombia; \\ hwpulidob@unal.edu.co \\ 2. Profesor Asociado, Instituto de Ciencias Naturales, Universidad Nacional de Colombia, Apartado 7495, Bogotá, D.C., \\ Colombia; mgandradec@unal.edu.co \\ 3. Museo de Zoología, Facultad de Ciencias, UNAM Apdo. Postal 70-399. México 04510 D.F. México; \\ jlb@hp.fciencias.unam.mx \\ * Autor de correspondencia
}

Recibido 15-IV-2009. Corregido 27-VIII-2009. Aceptado30-IX-2009.

\begin{abstract}
The taxonomy and distribution of Nathalis (Lepidoptera: Pieridae) in Colombia. In Colombia, Nathalis has two described species: $N$. iole and $N$. plauta. Previous authors did not make detailed descriptions of its distribution in meridional regions and failed to differentiate both species based on genitalic characters. Some wing marks have been enough to separate them, but co-specificity was a possibility. They inhabit Colombia above $2000 \mathrm{~m}$ in the paramo, and have a vicariant distribution from the remaining population of $N$. iole in the Antillean and Central and North America. An analysis focused on male and female genitalia, as well as the wing pattern of more than 100 specimens from the Colombian Andes (Sierra Nevada de Santa Marta and Sierra de Perijá) and Mexico, indicates that the two species differ in their genitalia, and considering their allopatric distribution, we support the specific distinction of $N$. iole and $N$. plauta. We describe a new endemic subspecies found exclusively in the paramo above $3000 \mathrm{~m}$, an area where other endemics occur. It has phenotypic plasticity related to environmental factors. Rev. Biol. Trop. 58 (1): 273-285. Epub 2010 March 01.
\end{abstract}

Key words: endemism, Pieridae, peripatric distribution, Andes, paramo.

El género Nathalis fue propuesto por Boisduval (1836) y actualmente incluye dos especies descritas, Nathalis iole Boisduval, 1836 y Nathalis plauta Doubleday, 1847 (Lamas 2004, Le Crom et al. 2004). Pertenece a la subfamilia Coliadinae (Lepidoptera: Pieridae) (Ehrlich 1958, Ackery et al. 1999), que es de distribución cosmopolita, aunque Klots (1931) lo ubicó en Rhodocerini (Pierinae) que equivale a los Coliadinae actuales. A nivel global la familia posee 1100 especies, dispuestas en la actualidad en 98 taxones (83 géneros y 15 subgéneros) (Braby et al. 2006). En Colombia la familia contiene 35 géneros, 147 especies y 223 subespecies, y los Coliadinae incluyen 12 géneros, 35 especies y 36 subespecies (Andrade-C, en prensa), la proporción mayor de especies de esta subfamilia se encuentra en tierras tropicales, en donde se supone se originó (Braby et al. 2006). Aunque al parecer es un grupo parafilético y todavía están por resolverse las relaciones del grupo con las otras tres subfamilias dentro de los piéridos, se asume que Coliadinae es el grupo hermano de Pierinae (Braby et al. 2006).

Nathalis iole es una especie de distribución amplia en el sur de la región Neártica, México, América Central y parte de las Antillas, vuela desde Estados Unidos hasta el norte de Colombia en la Sierra Nevada de Santa Marta, presentando varias poblaciones disyuntas entre las cuales no ocurre flujo genético (Douglas \& Grula 1978, Takahashi 1976, Torres 1986). 
En toda el área de distribución presenta varias formas estacionales, lo cual se ha citado como una respuesta fisiológica a los cambios de temperatura del medio, facilitando la ampliación de su distribución geográfica y de su hábitat (Douglas \& Grula 1978). Esta plasticidad fenotípica reflejada en la variación de la coloración también es una función de los cambios en el fotoperíodo (Douglas \& Grula 1978, Shapiro 1976, 1980), siendo las formas de invierno melánicas, y las de verano claras, en países con estacionalidad.

Nathalis plauta es endémica de la Cordillera Oriental de Colombia, en zonas sobre los $2000 \mathrm{~m}$ de altitud; es una especie con coloración más estable, ya que en el trópico la temperatura no fluctúa mucho a lo largo del año. En Colombia, ambas especies son típicas de áreas abiertas, como pajonales y páramos, donde se alimentan especialmente de plantas de la familia de las compuestas (Le Crom et al. 2004).

Lamas (2004) reconoce únicamente a $N$. iole y N. plauta; las demás especies, formas, aberraciones y variedades que históricamente fueron descritas se encuentran sinonimizadas, debido a que los diferentes fenotipos pertenecen a formas estacionales cuyas características están presentes en Nathalis y en otros géneros de Pieridae (Douglas \& Grula 1978, Shapiro 1976, 1977).

A Nathalis pertenecen mariposas pequeñas $(<30 \mathrm{~mm}$.) similares a Colias; en el campo se confunden por su vuelo, aunque Nathalis es más lenta y de vuelo más local que Colias. Klots (1931) y Le Crom et al. (2004) describieron las características más relevantes del género, además de algunos de los caracteres diagnósticos de las dos especies presentes en Colombia. Dentro de las plantas huésped de $N$. iole se han citado especies de los géneros Dyssonia, Bidens, Tagetes, Stellaria, Helenium y Erodium para Estados Unidos y América Central (Le Crom et al. 2004), mientras que para Colombia se han registrado en plantas de Cissia y Bidens (Torres 1986).

Debido a disyunción biogeográfica de las poblaciones de $N$. iole colombiana, su circunscripción altitudinal (estenotópica) y algunas marcas de las alas, se examinaron y compararon detalladamente estas poblaciones con otras, especialmente de México y Estados Unidos.

\section{MATERIALES Y MÉTODOS}

Los ejemplares de $N$. iole fueron recolectados entre los años 2005-2007 en páramos de la Serranía del Perijá, en el intervalo 3000-3700m de altitud. Otros ejemplares de $N$. iole de la Sierra Nevada de Santa Marta fueron encontrados después y examinados en el Instituto de Ciencias Naturales de Colombia en Bogotá (ICN-MHN-L). Los datos de distribución de $N$. plauta fueron tomados de varias colecciones entomológicas en Colombia que se citan adelante. Los genitales fueron extraídos usando un tratamiento de $\mathrm{KOH}$ caliente durante $10 \mathrm{minu}$ tos y observados con un estereoscopio Zeiss Stemi 2000-C; después fueron conservados en viales con alcohol y glicerina. Las fotografías de los genitales se realizaron con una cámara digital Nikon Coolpix 8700. Se usó el programa AxioVision 3.1 para la medición de genitales y Photoshop CS2 para componer las imágenes. Las descripciones de los colores en Photoshop CS2 siguen el modelo RGB por ser un espacio de color independiente del equipo.

La terminología de los genitales se basa en Klots (1931) y la del patrón alar se basa en Miller (1970) (Fig. 1). La nomenclatura siguiente y abreviaturas son usadas a través del texto:

AA: Ala anterior. AAD: Ala anterior en vista dorsal. AAV: Ala anterior en vista ventral. AP: Ala posterior. APD: Ala posterior en vista dorsal. APV: Ala posterior en vista ventral. Las siglas o acrónimos para las colecciones son: ICN-MHN-L: Instituto de Ciencias Naturales, Colección de Lepidoptera, Universidad Nacional de Colombia, Bogotá, Colombia. CDMB: Colecciones Biológicas del Jardín Botánico Eloy Valenzuela, Bucaramanga, Colombia. MEUS: Museo Entomológico de la Universidad de la Salle, Bogotá, Colombia. JFLECROM: Colección de J.F. Le Crom, Bogotá, Colombia. 
La abreviatura Gen es para genitales y RGB el modelo de color basado sobre rojo, verde y azul.

\section{RESULTADOS}

Nathalis iole natalia Pulido, Andrade \& Llorente ssp. nov.

\section{English diagnosis of new taxon}

FW length: 14.0mm; it can be separated from the North American subspecies $(N$. iole iole), by the following characters: inner margin of the DFW with a black diffuse or not well defined patch from basal area to distal area, VFW costal margin without the deep yellow or orange patch characteristic of the subspecies from the North. The genitalia are not different from the nominotypic subspecies.
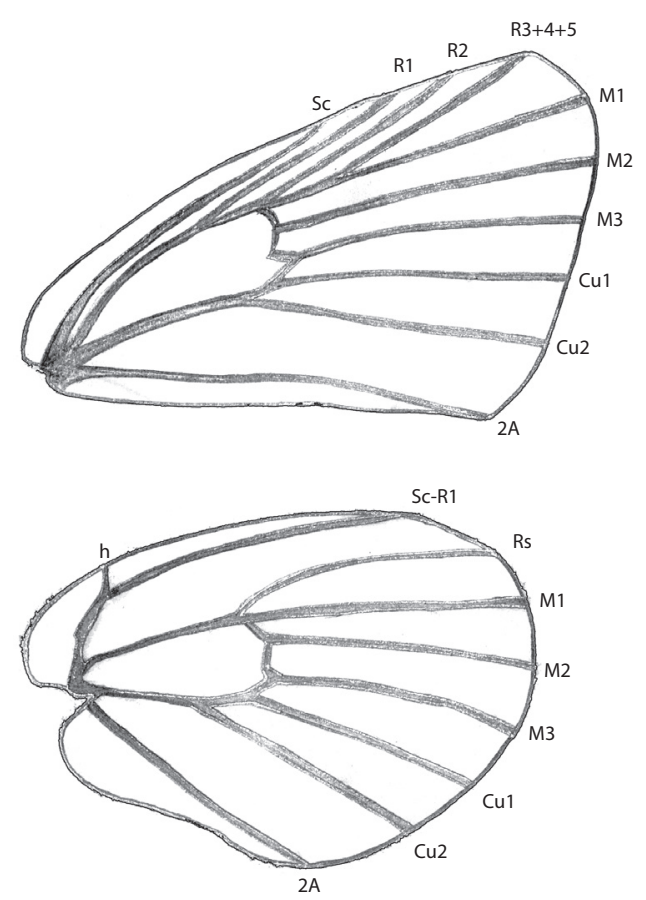

Fig. 1. Venación y nomenclatura de Nathalis iole natalia.

Fig. 1. Wing venation and nomenclature of Nathalis iole natalia.
Diagnosis: Longitud del AA: 14.0mm; puede ser separada de la subespecie norteamericana ( $N$. iole iole), por el siguiente conjunto de caracteres, margen interno del AAD con una mancha negra difusa o poco definida desde el área basal hasta el área discal, margen costal del AAV sin mancha amarilla intensa o naranja como en la subespecie del norte. Los órganos genitales no se diferencian de aquellos de la subespecie nominotípica.

HOLOTIPO: Colombia, La Guajira, Villanueva, Cerro El Pintao, 10 $20^{\circ}$ '0.5' N, $72^{\circ} 52^{\prime} 52^{\prime \prime} \mathrm{W}, 3130 \mathrm{~m}, 8$ de agosto de 2007, H. Pulido-B. Leg., HP 3045, Gen 1211, ICNMHN-L 21341.

ALOTIPO: Colombia, La Guajira, Villanueva, Cerro El Pintao, 10 $10^{\circ} 27^{\prime} 0.5^{\prime \prime} \mathrm{N}$, $72^{\circ} 52^{\prime} 52^{\prime}$ 'W, 3130m, 8 de agosto de 2007, H. Pulido-B. Leg., HP 3038, Gen 1210, ICNMHN-L 21349.

PARATIPOS: 5 machos y 1 hembra con la misma localidad que el holotipo: machos: HP 3023, Gen 1212, ICN-MHN-L 21360; HP 3044, Gen 1213, ICN-MHN-L 21348; HP 3035, Gen 1214, ICN-MHN-L 21344; HP 3021, ICN-MHN-L 22487; HP 3026, ICN-MHN-L 22484, HP 3034, ICN-MHN-L 22472; hembra: Colombia, Cesar, La Paz, San José del Oriente, Alto del Perijá, Finca Los Sauces, 10¹4'35' N, 7257'20" W, 3400m, 25 de Febrero de 2006, H. Pulido-B. Leg., Cesar, La Paz, San José del Oriente, Alto del Perijá, Finca Los Sauces, 10¹4'35" N, 7257'20" W, 3400m, 25 de Febrero de 2006, H. Pulido-B. Leg., HP 106, Gen 1215, ICN-MHN-L 19829; hembra, HP 89, Gen 1217, ICN-MHN-L 19833; macho, HP 92, ICN-MHN-L 19820; macho, HP 92, ICN-MHN-L 19823; macho, HP 73, Gen 1218, ICN-MHN-L 19827, macho, HP 87, Gen 1216, ICN-MHN-L 19828; macho, HP 72, Gen 1219, ICN-MHN-L 19831; hembra, Cesar, Manaure, El Cinco, Sabana Rubia, 10²1'48' norte, $72^{\circ} 55^{\prime} 32^{\prime \prime}$ oeste, 3200m, 25 de Febrero de 2007, H. Pulido-B. Leg., HP 802, ICN-MHN-L 19947; hembra, 2800m, HP 809, ICN-MHN-L 
19964; hembra, 3200m, HP 804, ICN-MHN-L 19970; hembra, 3155m, HP 673, Gen 1198, ICN-MHN-L 20004; hembra, 3176 m, HP 707, Gen 1208, ICN-MHN-L 20005; hembra, 3349m, HP 764, Gen 1207, ICN-MHN-L 20006; hembra, 3160m, HP 721, ICN-MHN-L 20020; hembra, 3165m, HP 727, ICN-MHN-L 20028; macho, 3200m, HP 805, ICN-MHN-L 19930; macho, HP 792, ICN-MHN-L 19934; macho, HP 803, ICN-MHN-L 19935; macho, HP 788, ICN-MHN-L 19940; macho, HP 793, ICN-MHN-L 19941; macho, HP 794, ICNMHN-L 19942; macho, HP 786, ICN-MHN-L 19943; macho, HP 798, ICN-MHN-L 19945; macho, HP 791, ICN-MHN-L 19949; macho, HP 795, ICN-MHN-L 19951; macho, HP 796, ICN-MHN-L 19952; macho, HP 801, ICNMHN-L 19954; macho, HP 800, ICN-MHN-L 19960; macho, HP 799, ICN-MHN-L 19963; macho, HP 785, ICN-MHN-L 19965; macho, HP 781, ICN-MHN-L 19966; macho, HP 806, ICN-MHN-L 19967; macho, HP 797, ICNMHN-L 19968; macho, HP 782, ICN-MHN-L 19971; macho, HP 712, Gen 1197, ICN-MHNL 19989; macho, HP 714, ICN-MHN-L 19990; macho, HP 718, Gen 1196, ICN-MHN-L 19991; macho, HP 716, Gen 1195, ICN-MHNL 19992; macho, HP 715, Gen 1194, ICNMHN-L 19993; macho, HP 711, Gen 1193, ICN-MHN-L 19994; macho, HP 717, Gen 961, ICN-MHN-L 19995; macho, HP 720, Gen 1191, ICN-MHN-L 19996; macho, HP 713, Gen 1190, ICN-MHN-L 19997; macho, HP 719, Gen 1189, ICN-MHN-L 19998; macho, HP 778, Gen 1188, ICN-MHN-L 19999; macho, 3300m, HP 776, Gen 1192, ICN-MHNL 20000; macho, 3000m, HP 681, Gen 963, ICN-MHN-L 20001; macho, 3673m, HP 759, ICN-MHN-L 20002; macho, 3300m, HP 675, Gen 1209, ICN-MHN-L 20003; macho, HP 696, Gen 1206, ICN-MHN-L 20007; macho, $3120 \mathrm{~m}$, HP 699, Gen 1205, ICN-MHN-L 20008; macho, HP 703, Gen 1204, ICN-MHNL 20009; macho, HP 698, Gen 1203, ICNMHN-L 20010; macho, HP 702, Gen 1202, ICN-MHN-L 20011; macho, HP 697, Gen 1201, ICN-MHN-L 20012; macho, HP 701, Gen 1200, ICN-MHN-L 20013; macho, HP
704, Gen 1199, ICN-MHN-L 20014; macho, HP 706, Gen 960, ICN-MHN-L 20015; macho, HP 710, Gen 964, ICN-MHN-L 20016; macho, HP 709, Gen 959, ICN-MHN-L 20017; macho, HP 777, ICN-MHN-L 20018; macho, HP 722, ICN-MHN-L 20019; macho, HP 680, ICNMHN-L 20021; macho, HP 738, ICN-MHN-L 20022; macho, HP 1113, ICN-MHN-L 20023; macho, HP 724, ICN-MHN-L 20025; macho, HP 725, ICN-MHN-L 20026; macho, HP 730, ICN-MHN-L 20027; macho, HP 732, ICNMHN-L 20029; macho, HP 705, ICN-MHN-L 20030; macho, HP 708, ICN-MHN-L 20031; macho, HP 676, ICN-MHN-L 20032; macho, HP 674, ICN-MHN-L 20033; hembra, La Guajira, Villanueva, Cerro El Pintao, 10²7'0.5" N, 72 52 '52" W, 3130m, 08 de Agosto de 2007, H. Pulido-B. Leg., HP 3038, Gen 1210, ICNMHN-L 21349; hembra, HP 3034, ICN-MHN-L 22472; macho, HP 3045, Gen 1211, ICN-MHNL 21341; macho, HP 3035, Gen 1214, ICNMHN-L 21344; macho, HP 3044, Gen 1213, ICN-MHN-L 21348; macho, HP 3023, Gen 1212, ICN-MHN-L 21360; macho, HP 3046, ICN-MHN-L 22465; macho, HP 3026, ICNMHN-L 22484; macho, HP 3021, ICN-MHN-L 22487; macho, Cesar, Valledupar, Meollaca, 30 de Junio de 1967, T. Sugimoto \& M. Takahashi. Leg., ICN-MHN-L 23775; macho, Río Donachui, 29 de Junio de 1967, T. Sugimoto \& M. Takahashi. Leg., ICN-MHN-L 23776.

\section{Macho}

Longitud del AA: 14.8mm (n=64) (Fig. 4A).

Cabeza: Palpos densamente cubiertos con escamas largas amarillas (R115, G113, B38) cuya longitud es dos veces el diámetro del ojo; longitud de la antena es la tercera parte del margen costal del AA, de color negro en vista dorsal y blanco en vista ventral, intersecciones de los segmentos de las antenas color blanco.

Tórax: Negro (R6, G14, B23) en vista dorsal, sin escamas, en vista ventral cubierto por densas escamas largas y amarillas (R151, G149, B79). 
Abdomen: Negro en vista dorsal con algunas escamas largas y amarillas, en vista ventral cubierto por pequeñas escamas amarillas (R151, G149, B79).

Genitales: Uncus largo $(0.58 \mathrm{~mm})$ y delgado, recto en su primera mitad y luego curvado en la segunda mitad; tegumen recto y corto $(0.28 \mathrm{~mm})$; gnathos ausente y valva alargada $(1.2 \mathrm{~mm})$ con dos procesos distales, uno medial bifurcado $(0.48 \mathrm{~mm})$ con dos ramificaciones que presentan numerosas espinas y otro en el extremo distal con dos espinas pequeñas en $\mathrm{su}$ base; saccus largo $(0.6 \mathrm{~mm})$ con extremo redondeado; aedeagus tubular $(1.65 \mathrm{~mm})$ con ondulaciones en vista lateral (Figs. 2C, D) y cornuti diminutos en el extremo distal a lo largo del margen lateral, cuando el pennis no está evertido (Fig. 2).
AA (Fig. 4A): Ápice redondeado, margen externo cubierto por cilia de color amarillo (R233, G240, B137) alternadas con otras de color negro (R95, G81, B42) en el extremo de las venas. AAD de color amarillo claro en el fondo (R228, G238, B115); ápice a manera de una mancha triangular negra (R51 G40, B34) en el área apical desde el espacio $\mathrm{M}_{3}-\mathrm{Cu}_{1}$ hasta el margen costal; banda delgada y gris en el margen costal, desde el área basal hasta la mancha apical; tres manchas grises difusas (R145, $\mathrm{G} 153, \mathrm{~B} 80$ ) en los espacios $\mathrm{M}_{3}-\mathrm{Cu}_{1}, \mathrm{Cu}_{1}-\mathrm{Cu}_{2}$ y $\mathrm{Cu}_{2}-2 \mathrm{~A}$, dos pequeñas manchas negras triangulares bien definidas en los extremos distales de las venas $\mathrm{Cu}_{1}$ y $\mathrm{Cu}_{2}$; banda negra desde el área basal a lo largo del margen anal que se hace difusa hacia el área media del ala. AAV de color amarillo claro en el fondo (R238, G249, B85), banda delgada y gris en el margen

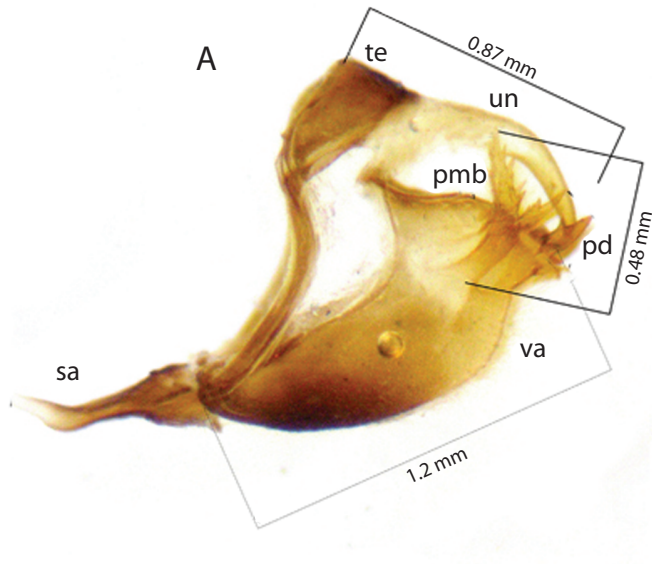

ae

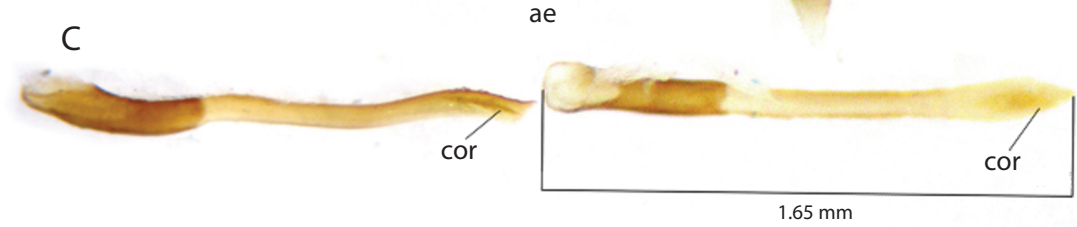

B

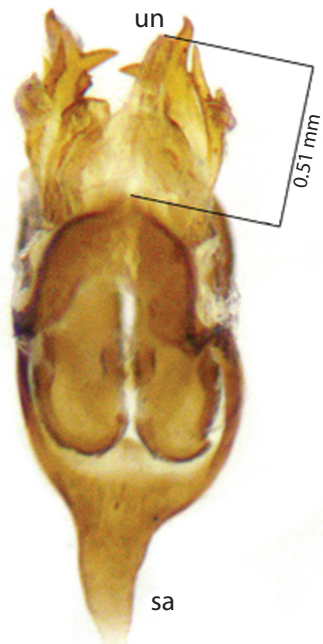

$1 \mathrm{~mm}$

Fig. 2. Órgano genital masculino de Nathalis iole natalia. A. Vista lateral, B. Vista Dorsal, C. Aedeagus en vista lateral, D. Aedeagus en vista dorsal; ae: aedeagus; un: uncus; te: tegumen; sa: saccus; va: valva; pmb: proceso medial bifurcado de la valva; pd: proceso distal de la valva; cor: cornuti.

Fig. 2. Male genitalia of Nathalis iole natalia. A. lateral view, B. dorsal view, C. Aedeagus (lateral view), D. Aedeagus (dorsal view); ae: aedeagus; un: uncus; te: tegumen; sa: saccus; va: valva; pmb: medial bifurcated process of the valva; pd: distal process of the valva; cor: cornuti. 
costal, desde el área basal hasta la mancha apical; ápice triangular difuso y las dos pequeñas manchas triangulares en las venas $\mathrm{Cu}_{1}$ y $\mathrm{Cu}_{2}$ en las mismas posiciones pero más difusas (R167, G175, B89) que en AAD, mientras que las tres manchas en los espacios $\mathrm{M}_{3}-\mathrm{Cu}_{1}$, $\mathrm{Cu}_{1}-\mathrm{Cu}_{2}$ y $\mathrm{Cu}_{2}-2 \mathrm{~A}$ son negras y bien definidas (R61, G55, B31); una mancha negra pequeña y alargada en $\mathrm{m}_{1}-\mathrm{m}_{2}$. AP: Ala redondeada con margen anal recto, margen externo cubierto con escamas amarillas como en AA. APD de color amarillo claro en el fondo (R220, G229, B86), con el área basal negra; venas $\mathrm{M}_{1}, \mathrm{M}_{2}, \mathrm{M}_{3}, \mathrm{Cu}_{1}$ y $\mathrm{Cu}_{2}$ delineadas de negro en la mitad distal, banda negra a lo largo del margen costal con un parche androconial. APV de color de fondo gris verdoso (R167, G172, B105) con escamas amarillas y negras esparcidas por toda la superficie del ala; celda discal con una mancha amarilla pequeña (R212, G217, B99); presenta una banda amarilla (R243, G250, B144) postdiscal que se extiende de manera irregular desde el margen costal hasta $\mathrm{M}_{3}$. Área submarginal del termen ligeramente más clara.

\section{Hembra}

Longitud del AA: 14.1mm ( $\mathrm{n}=12)$ (Figs. 3, 4B). Casi igual que el macho pero de alas más alargadas y redondeadas. En vista dorsal, el color general es más pálido, pero en vista ventral el color de fondo es un poco más oscuro.

Genitales (Fig. 3): Papillae anales amplias con sedas largas; ductus bursae fuertemente esclerosado en el primer tercio, membranoso en la parte media y fuertemente esclerosado en la parte distal; corpus bursae redondeado y esclerosado con signa que se compone de dos espinas, una larga y otra corta.

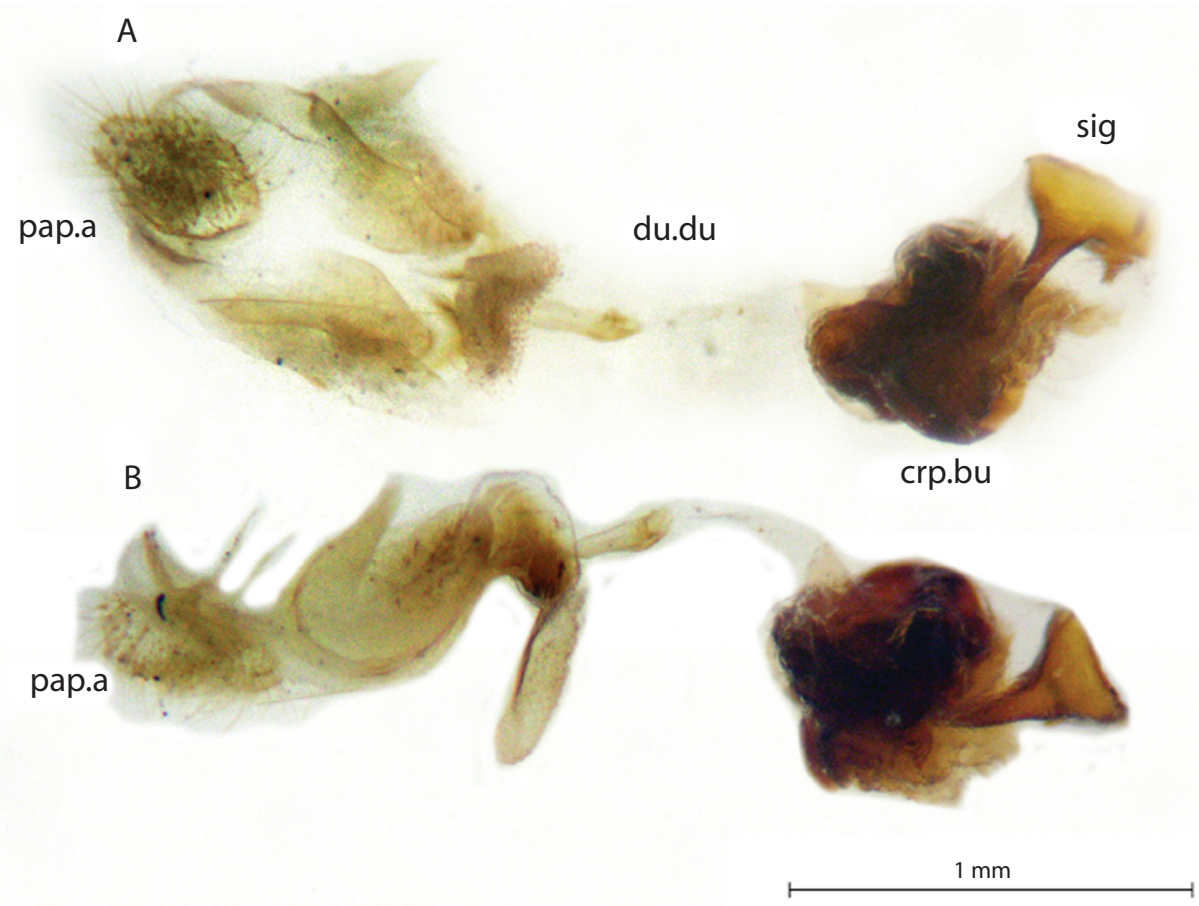

Fig. 3. Órgano genital femenino de Nathalis iole natalia. A. Vista dorsal, B. Vista lateral; pap.a: papillae analis; du.bu: ductus bursae; crp.bu: corpus bursae; sig: signa.

Fig. 3. Female genitalia of Nathalis iole natalia. A. Dorsal view, B. Lateral view; pap.a: papillae analis; du.bu: ductus bursae; crp.bu: corpus bursae; sig: signa. 
AA (Fig. 4B): Color dorsal de fondo ligeramente más pálido (R235, G241, B103) que en el macho, el patrón de manchas en AAD es similar al macho pero el negro es más pálido (R56, G56, B27); la banda negra a lo largo del margen anal es más difusa y más delgada. AAV de color amarillo más oscuro (R242, G246, B61) que en el macho y con el mismo patrón de manchas, aunque éstas son un poco más extendidas o mayores. AP: De color dorsal algo más pálido que en el macho al igual que en AAD. APD con una mancha negra en $\mathrm{M}_{1}-\mathrm{M}_{2}$, en el área postdiscal. APV de color general gris amarillento más oscuro que en el macho, con el mismo patrón de manchas excepto por una banda negra discontinua en el área postdiscal que se extiende desde $\mathrm{M}_{1}$ hasta $2 \mathrm{~A}$.

Los estados inmaduros y plantas huésped son desconocidos.
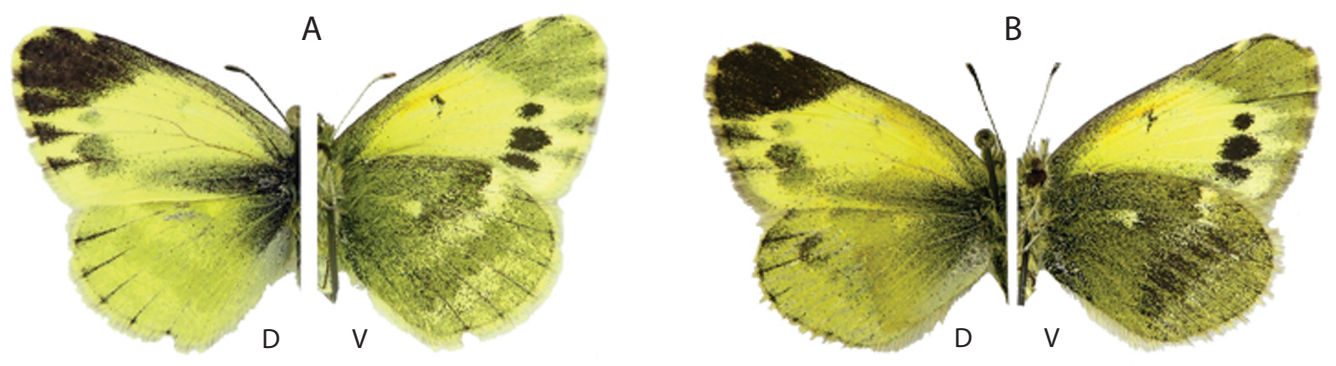

Fig. 4. Adultos de Nathalis iole natalia, A. Holotipo, B. Alotipo. Ambos de Villanueva (La Guajira, Colombia). D: Vista dorsal, V: Vista ventral.

Fig. 4. Adults of Nathalis iole natalia, A. Holotype, B. Allotype. Both from Villanueva (La Guajira, Colombia). D: Dorsal view; V: Ventral view.

Etimología: Dedicamos el nombre de esta subespecie a la hija de Jorge Llorente, el nombre sin latinizar.

Distribución y ecología: Solamente se conoce para el área norte de la Serranía del Perijá, volando en zonas de páramos sobre 3000m y en la Sierra Nevada de Santa Marta en la vertiente sur (Fig. 7). Las poblaciones son abundantes localmente, volando cerca del suelo entre la vegetación del páramo y por caminos despejados.

La población del Perijá y la de la Sierra Nevada de Santa Marta están aisladas y disyuntas por $100 \mathrm{~km}$; aunque no hay evidencia de que ocurra flujo genético entre las poblaciones de ambas áreas, es posible que eventualmente individuos de un lado y otro sean dispersados pasivamente por el viento.
Su distribución en un gradiente altitudinal es peculiar, pues en Norteamérica y México llega a ser común en tierras bajas, mientras que en la Serranía del Perijá solamente se encontró por encima de los $3000 \mathrm{~m}$, en ambientes de páramo. Si se tienen en cuenta los muestreos intensivos y sistemáticos en tierras más bajas, se concluye que $N$. iole natalia es una subespecie local con distribución restringida en los páramos de la Serranía del Perijá y la Sierra Nevada de Santa Marta. Aunque en esta última se ha citado como el límite inferior de su distribución los 1300m de altitud (Takahashi 1976), colectores sucesivos no se han vuelto a encontrar a esta altitud (Winhard com. pers.); tal vez una limitante en la distribución altitudinal sea la presencia de las plantas huésped. Los imagos de $N$. iole natalia son mariposas resistentes a las condiciones frías del ecosistema, de tal 
manera que era la única especie que volaba en las mañanas frías y nubladas de enero, cuando la temperatura ambiental era apenas de $10^{\circ} \mathrm{C}$.

\section{DISCUSIÓN}

Variaciones poblacionales (Fig. 5). $N$. iole es una especie que exhibe variación morfológica en algunas estructuras de los genitales masculinos. Al considerar tales variaciones, se examinaron con detalle numerosos individuos provenientes de México (Veracruz, Oaxaca y Valle de México), de la Sierra Nevada de Santa Marta y de tres localidades en la Serranía del Perijá, tanto en el diseño alar como en los genitales masculinos y femeninos.

El patrón alar no presenta mayor variación excepto por las características previamente mencionadas para la subespecie colombiana. No existen diferencias estacionales, ya que en Colombia no se presenta estacionalidad acentuada, pues la temperatura a lo largo del año es relativamente constante. Mientras que en México y los Estados Unidos, las diferencias climáticas de las estaciones tienen un efecto fenotípico en las poblaciones de $N$. iole (Douglas \& Grula 1978) y de otros piéridos (Shapiro 1968, 1973, 1975, 1977, Oliver 1970, Kingsolver 1987), lo que se ve reflejado en cambios de diseño o coloración. Las poblaciones colombianas son estables en cuanto a diseño y coloración durante todo el año, debido a la estabilidad climática, aunque es posible que mantengan latente esta plasticidad fenotípica de sus homólogos boreales. Debido a las bajas temperaturas del páramo los individuos de $N$. iole natalia presentan un incremento en la melanización ventral en comparación a las poblaciones mexicanas, por ello son similares a las poblaciones invernales del hemisferio norte. Esto puede tener importancia fisiológica en la termoregulación.

Los órganos genitales presentan variación entre individuos de una misma población, sobre todo en las "espinas" presentes en las ramas del proceso medial bifurcado de la valva; tales "espinas" pueden ser numerosas, escasas o no se presentan en algunos individuos.
Algunos especímenes incluso llegan a presentar asimetría bilateral en los genitales por razón de tales procesos espiniformes. La asimetría en la naturaleza se da en caracteres que parecen no tener presión selectiva ni significado evolutivo aparente, por lo que estas variaciones tanto en las poblaciones de México como en las de Colombia nos llevan a concluir que tales estructuras en los órganos genitales masculinos son poco informativos para diferenciar subespecies en Nathalis iole.

Genitales masculinos de Nathalis iole y Nathalis plauta (Fig. 6, Cuadro 1). Se ha criticado mucho el valor taxonómico de los caracteres en los órganos genitales en algunos géneros de Pieridae (Fruhstorfer 1908, Klots 1931, Bollino \& Costa 2007), debido a que la variación de los individuos puede ser amplia en algunas estructuras que normalmente son de valor taxonómico. En particular, Klots (1931) afirmó no encontrar caracteres estables para diferenciar $N$. iole de $N$. plauta (ver adelante); sin embargo, durante la observación minuciosa y comparación detallada de una extensa cantidad de material de $N$. iole (más de 100 especímenes) proveniente de varias localidades de México y de la Serranía del Perijá, Colombia y comparado con los órganos genitales de $N$. plauta (más de 20 especímenes) de varias localidades de la Cordillera Oriental colombiana, encontramos diferencias constantes en algunos caracteres de los órganos genitales que van más allá de la simple variación poblacional, lo que permite usarlos para separar las especies del género (Cuadro 1). No obstante la declaración de Klots (1931: 141-142) "Como un ejemplo de esto, Nathalis servirá de manera excelente. El autor ha sido incapaz de encontrar cualquier carácter estable para diferenciar iole y plauta por genitales. Por otro lado, los genitales de estas dos especies son tan diferentes de los de cualquier otro Pieridae que se consideran caracteres genéricos importantes..." Y como ha demostrado este estudio en acuerdo con Klots (1931: 142) "Las creencias con respecto a la poca fiabilidad de los genitales masculinos en Pieridae, debido a las variaciones 

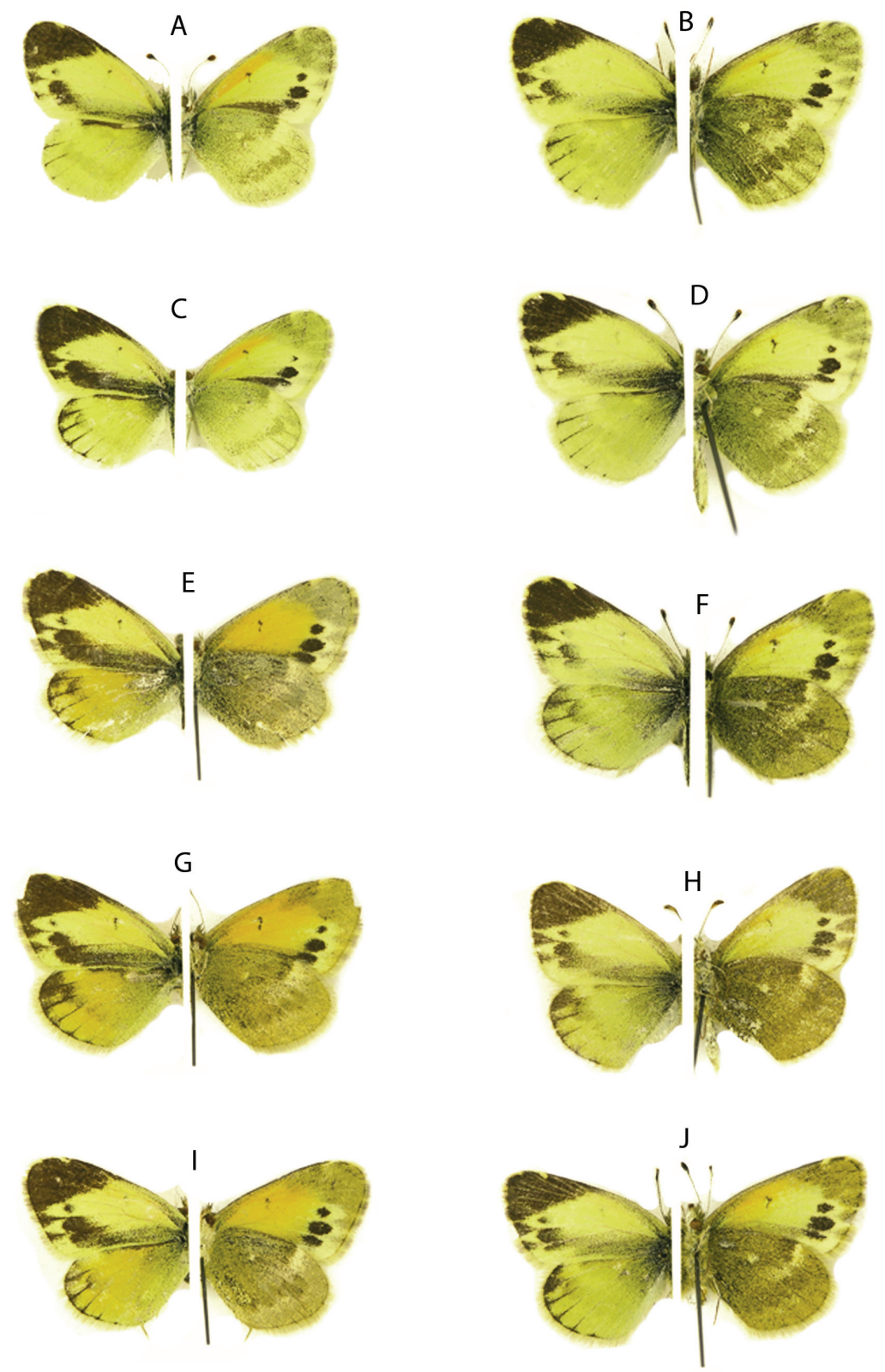

Fig. 5. Variación morfológica entre las subespecies de Nathalis iole. Columna de la izquierda: N. iole iole. Columna de la derecha: N. iole natalia. A. Macho, México, Zumpahuacan, 1600 m; B. Macho, Colombia, El Pintao, 3130 m; C. Macho, México, Malinalco, 1670 m; D. Macho, Colombia, Manaure, 2940; E. Hembra, México, Contreras, 2800 m; F. Hembra, Colombia, El Pintao, 3130 m; G. Hembra, México, Contreras, 2800 m; H. Hembra, Colombia, Manaure, 2800 m; I. Hembra, México, Contreras, 2800 m; J. Hembra, Colombia, Manaure, 3176 m.

Fig. 5. Phenotypic plasticity in the subspecies of Nathalis iole. Left: N.iole iole. Right: N. iole natalia. A. Male, Mexico, Zumpahuacan, 1600 m; B. Male, Colombia, El Pintao, 3130 m; C. Male, Mexico, Malinalco, 1670 m; D. Male, Colombia, Manaure, 2940; E. Female, Mexico, Contreras, 2800 m; F. Female, Colombia, El Pintao, 3130 m; G. Female, Mexico, Contreras, 2800 m; H. Female, Colombia, Manaure, 2800 m; I. Female, Mexico, Contreras, 2800 m; J. Female, Colombia, Manaure, $3176 \mathrm{~m}$. 


\section{CUADRO 1}

Comparación de algunas estructuras genitales entre Nathalis iole $y$ N. plauta

TABLE 1

Comparison between Nathalis iole and N. plauta genitalia

\section{Nathalis plauta}

Apariencia general más grande

Uncus con leve curvatura ventral

Margen superior de la valva ondulado

Vinculum recto

\section{Saccus recto}

Proceso distal de la valva con una única "espina” grande en la base

Aedeagus ligeramente más amplio

Apertura proximal del aedeagus con ensanchamiento en el medio

Cornuti en aedeagus terminan poco antes del extremo distal Aedeagus recto en vista lateral

Signa del corpus bursae con una sola "espina" (larga)

\section{Nathalis iole}

Apariencia general más pequeña

Uncus curvado ventralmente

Margen superior de la valva casi recto

Vinculum convexo

Saccus semicurvo

Proceso distal de la valva con dos pequeñas "espinas" en la base

Aedeagus ligeramente más delgado

Apertura proximal del aedeagus alargada

Cornuti en aedeagus terminan en el extremo distal

Aedeagus ligeramente curvo en vista lateral

En la hembra, signa del corpus bursae con dos "espinas": una larga y otra corta

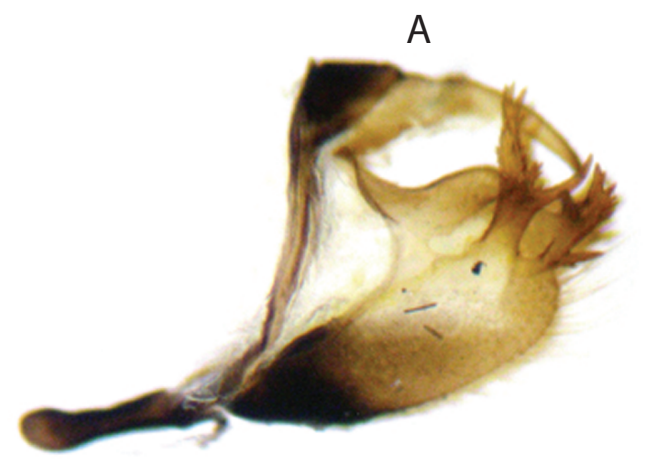

B
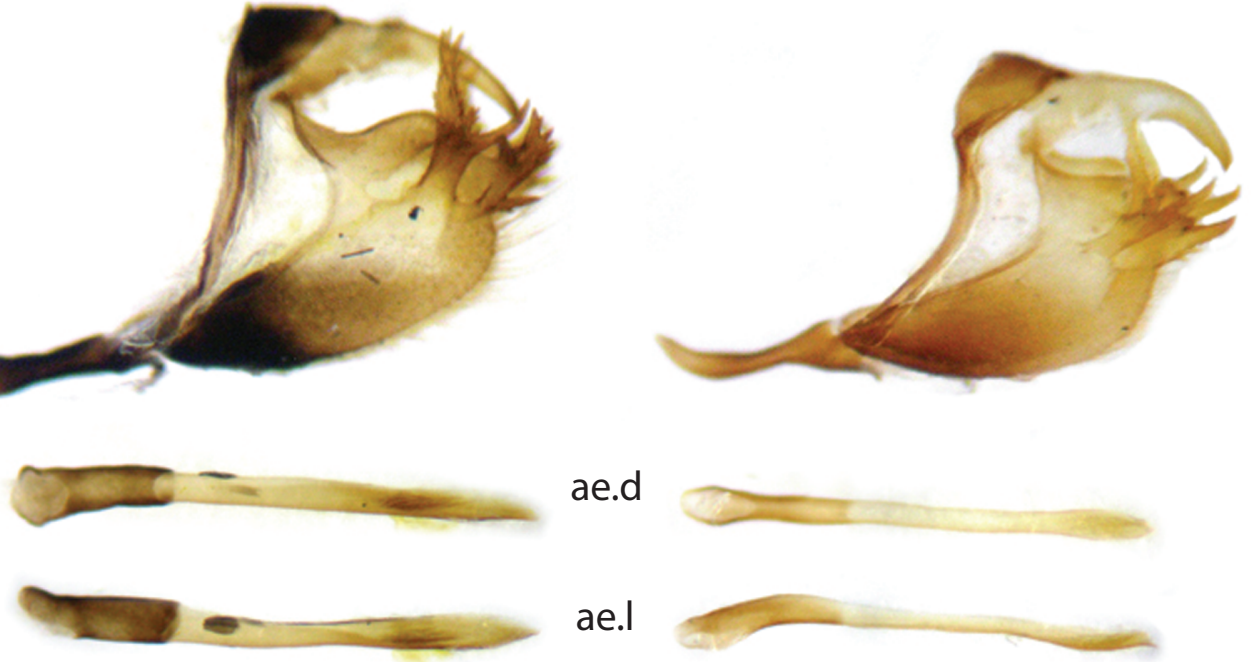

ae.d

ae.l

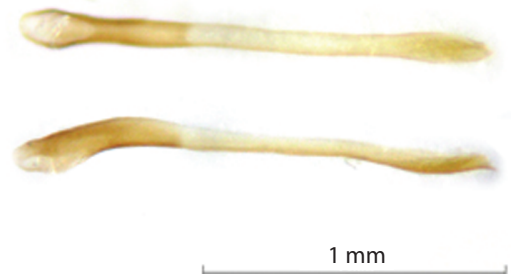

Fig. 6. Órganos genitales masculinos de las especies de Nathalis: A. Nathalis plauta, B. Nathalis iole natalia. ae.d: aedeagus en vista dorsal; ae.l: aedeagus en vista lateral.

Fig. 6. Male genitalia of the Nathalis species: A. Nathalis plauta, B. Nathalis iole natalia. ae.d: aedeagus (dorsal view); ae.l: aedeagus (lateral view). 
individuales, están basadas probablemente en lo siguiente: a. El estudio de muy pocos especímenes. $b$. De principio, una tendencia a esperar demasiado de los genitales. c. Técnica defectuosa, irregular o descuidada, o el uso de especímenes mal preservados, lo que resulta en una distorsión de los genitales". Al observar las indicaciones previas, haciendo observaciones y comparaciones sumamente cuidadosas de los genitales masculinos de varias decenas de ejemplares, encontramos los caracteres que se muestran en el cuadro 1, los que consideramos suficientes para satisfacer la separación específica de estas especies disyuntas, solucionando un problema añejo: sustentar la separación de ambas especies sobre bases firmes.

\section{Distribución de Nathalis en Colombia.} Nathalis plauta vuela en una pequeña franja andina desde Bogotá hasta el Norte del Departamento de Santander en Colombia, está restringida al flanco occidental de la Cordillera Oriental; mientras que $N$. iole natalia -disyunta a esta distribución- vuela de manera restringida en un sector reducido de la parte norte de la Serranía del Perijá y la Sierra Nevada de Santa Marta (Fig. 7). Ninguna de las especies se encontró en localidades intermedias entre Perijá y Santander, debido tal vez a que la Serranía

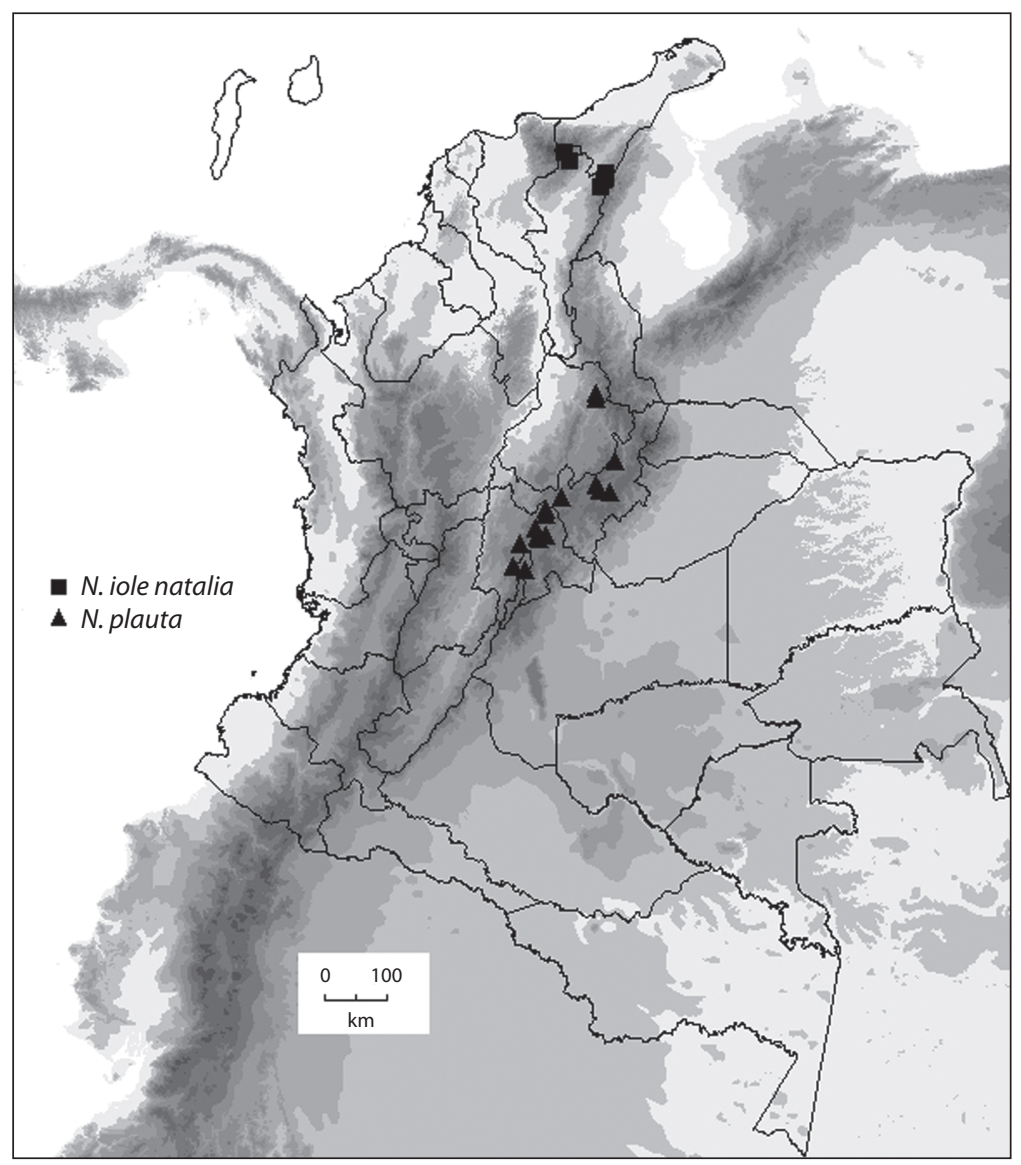

Fig. 7. Distribución del género Nathalis en Colombia.

Fig. 7. Distribution of the genus Nathalis in Colombia. 
de Perijá se separa de la Cordillera Oriental por la depresión Río de Oro a 1300m de altitud y ambas especies vuelan por arriba de los $2000 \mathrm{~m}$ de altitud; esta depresión tiene un papel de barrera fisiográfica y ecológica, en consecuencia no hay evidencia de hibridación natural.

Desde los trabajos de Adams (1973) y Adams \& Bernard (1977) se ha hecho hincapié en el aislamiento y endemismo de los Rhopalocera en la Sierra Nevada de Santa Marta; recientemente el trabajo de Purser (2009) destaca el área como zona de endémicos, el presente trabajo apoya tal hipótesis. Respecto a la Serranía del Perijá, los trabajos de Adams \& Bernard (1979) y Pulido \& Andrade (2007) señalan que es un área de endemismo, especialmente las zonas de páramos. El endemismo de Nathalis iole natalia confirma lo señalado por los autores mencionados.

\section{AGRADECIMIENTOS}

Agradecemos a Orlando Rangel y al grupo de Biodiversidad y Conservación del Instituto de Ciencias Naturales de la Universidad Nacional de Colombia, así como a la Corporación Autónoma Regional del Cesar CORPOCESAR, por financiar las expediciones a la Serranía del Perijá. A Angel Viloria y Walter Winhard por comentarios previos sobre la variación de las poblaciones colombianas de $N$. iole con respecto a las poblaciones de México y Norteamérica. Gracias también al proyecto TABDP por facilitar la cámara para la toma de fotografías. A Diana Caballero y Gustavo Torres, al Jardín Botánico Eloy Valenzuela (CDMB) y a Jean François Le Crom por facilitar datos sobre la distribución de N. plauta. A Arthur Shapiro por comentarios previos sobre la distribución y origen del género. A Nadja Grote por el dibujo de la venación de las alas. A Armando Calvano de la Fundación Prosierra Nevada de Santa Marta, por la información suministrada sobre las localidades de la Sierra Nevada de Santa Marta. A Blanca Claudia Hernández por los ejemplares de Nathalis de Malinalco. A Armando Luis y Marisol Trujano por los ejemplares del Valle de México, Veracruz y Oaxaca (México) de
Nathalis iole. Jorge Llorente agradece a los proyectos PAPIIT IN 203509 y CONACyT 83237, por su ayuda complementaria en un par de viajes a Bogotá. Jimena Castro nos auxilió en distintos borradores de este artículo y a leer críticamente el manuscrito.

\section{RESUMEN}

En Colombia, Nathalis comprende dos especies descritas: $N$. iole y $N$. plauta. Autores previos no describieron detalladamente su distribución meridional y fallaron en la diferenciación de ambas especies basado en sus caracteres genitales. Algunos caracteres de su diseño alar habían sido suficientes para separarlas, pero su co-especificidad pudo ser una posibilidad. Estas habitan en Colombia, por arriba de los $2000 \mathrm{~m}$ en el páramo, y tienen una distribución vicariante desde poblaciones remanentes de $N$. iole en las Antillas y en Centro y Norteamérica. Un análisis de la variación de los genitales masculinos y femeninos, así como del diseño alar de más de 100 especímenes de los Andes Colombianos (Sierra Nevada de Santa Marta y Sierra de Perijá) y México, indica que las dos especies difieren en sus genitales, y considerando su distribución alopátrida, nosotros confirmamos la distinción específica entre $N$. iole y $N$. plauta. Describimos una nueva subespecie endémica encontrada exclusivamente en el páramo por encima de los $3000 \mathrm{~m}$, un área en donde se dan otros endemismos. Las especies de Nathalis presentan plasticidad fenotípica debida a factores ambientales.

Palabras clave: Endemismo, Pieridae, distribución peripátrida, Andes, páramo

\section{REFERENCIAS}

Adams, M.J. 1973. Ecological zonation and the butterflies of the Sierra Nevada de Santa Marta, Colombia. J. Nat. Hist. 7: 699-718.

Adams, M.J. \& G.I. Bernard. 1977. Pronophiline butterflies (Satyridae) of the Sierra Nevada de Santa Marta, Colombia. Syst. Ent. 2: 263-281.

Adams, M.J. \& G.I. Bernard. 1979. Pronophiline butterflies (Satyridae) of the Serranía de Valledupar, ColombiaVenezuela Border. Syst. Ent. 4: 95-118.

Andrade-C., M. G. 2008. Análisis de la distribución y lista anotada de las especies de la familia Pieridae (Lepidoptera: Papilionoidea) para Colombia. Rev. Acad. Colomb. Cienc. En prensa.

Ackery, P.R., R. de Jong \& R.J. Vane-Wright. 1999. The butterflies: Hedyloidea, Hesperioidea and 
Papilionoidea, p. 263-3000. In N.P. Kristensen. Lepidoptera, moths and butterflies. Vol. 1: Evolution, systematics and biogeography. Hadbuch der Zoologie. Vol. IV. Arthropoda: Insecta Teilband 35. Walter de Gruyter, Berlín Alemania.

Bollino, M. \& M. Costa. 2007. An illustrated annotated checklist of the species of Catasticta (s.1.) Butler (Lepidoptera: Pieridae) of Venezuela. Zootaxa 1469: 1-42.

Braby, M.F., R. Vila \& N.E. Pierce. 2006. Molecular phylogeny and systematics of the Pieridae (Lepidoptera: Papilionoidea): higher classification and biogeography. Zool. J. Linn. Soc. 147: 239-275.

Douglas, M.M. \& J. Grula. 1978. Thermoregulatory adaptations allowing ecological range expansion by the pierid butteffly, Nathalis iole Boisduval. Evolution 32: $776-783$

Ehrlich, P.R. 1958. The comparative morphology, phylogeny and higher classification of the butterflies (Lepidoptera: Papilionoidea). Univ. Kans. Sci. Bull. 39: $305-370$

Fruhstorfer, H. 1908. Neues über die Genitalorgane der Pieriden. Entomologische Zeitschrift. 22: 198-207.

Kingsolver, J.G. 1987. Evolution and coadaptation of thermoregulatory behavior and wing pigmentation pattern in pierid butterflies. Evolution 41: 472-490.

Klots, A. B. 1931-1932. A generic revision of the Pieridae (Lepidoptera): Together with a study of the male genitalia. Entomol. Am. 12: 139-242.

Lamas, G. (Ed.) 2004. Atlas of Neotropical Lepidoptera Checklist: part 4A Hesperioidea-Papilionoidea. Association for Tropical Lepidoptera, Florida, EEUU.

Le Crom, J.F., J. Llorente-Bousquets, L.M. Constantino \& J.A. Salazar. 2004. Mariposas de Colombia. Vol. 2. Pieridae. Carlec, Bogotá, Colombia.

Miller, L.D. 1970. Nomenclature of wing veins and cells. J. Res. Lepid. 8: 37-48.

Oliver, C.G. 1970. The environmental regulation of seasonal dimorphism in Pieris napi oleracea (Pieridae). J Lep. Soc. 24: 77-81.
Pulido-B., H.W. \& M.G. Andrade-C. 2007. Mariposas de las partes altas de la Serranía de Perijá, p. 235-248. In J.O. Rangel-Ch. Colombia Diversidad Biótica V: La alta montaña de la Serranía de Perijá. Instituto de Ciencias Naturales, Bogotá, Colombia.

Shapiro, A.M. 1968. Photoperiodic induction of vernal phenotype in Pieris protodice Boisduval. Wassmann J. Biol. 26: 137-149.

Shapiro, A.M. 1973. Photoperiodic control of seasonal polyphenism in Pieris occidentalis Reakirt. Wassmann. J. Biol. 31: 291-299.

Shapiro, A.M. 1975. Photoperiodic control of development and phenotype in a subartic population of Pieris occidentalis (Lep:Pieridae).Can. Entomol. 107: 775-779.

Shapiro, A. M. 1976. Seasonal Polyphenism, In Hecht, Steere, Wallace. Evolutionary Biology. Vol. 9. Plenum, Nueva York, EEUU.

Shapiro, A.M. 1977. Phenotypic induction in Pieris napi L.: role of temperature and photoperiod in a coastal Californian population. Ecol. Entomol. 2: 217-224.

Shapiro, A.M. 1980. Physiological and Developmental Responses to Photoperiod and Temperature as Data in Phylogenetic and Biogeographic Inference. Syst. Zool. 29: 335-341.

Takahashi, M. 1976. A list of the pieridae, collected in the Sierra Nevada de Santa Marta and the vicinity, Colombia, South America. Bull. Japan Entomol. Acad. 10: 13-25.

Torres, J.A. 1986. Contribución al estudio faunístico de los piéridos de Colombia (Lepidoptera: Pieridae). Tesis de Maestría, ICA, Universidad Nacional de Colombia, Bogotá, Colombia.

\section{REFERENCIA DE INTERNET}

Purser, B. 2009. Butterflies of the Sierra Nevada de Santa Marta, Colombia (Consultado: 15 febrero 2009, http://www.lepido-france.fr/2009/03/butterflies-ofthe-sierra-nevada-de-santa-marta-colombia/). 
